\title{
Unforeseen swimming and gliding mode of an insect gut symbiont, Burkholderia sp. RPE64, with wrapping of the flagella around its cell body
}

\author{
Yoshiaki Kinosita $\mathbb{D}^{1,3} \cdot$ Yoshitomo Kikuchi $^{2} \cdot$ Nagisa Mikami ${ }^{1} \cdot$ Daisuke Nakane $\mathbb{D}^{1} \cdot$ Takayuki Nishizaka $^{1}$
}

Received: 9 March 2017 / Revised: 9 October 2017 / Accepted: 24 October 2017 / Published online: 21 December 2017

(c) The Author(s) 2018, under exclusive licence to the International Society for Microbial Ecology

\begin{abstract}
A bean bug symbiont, Burkholderia sp. RPE64, selectively colonizes the gut crypts by flagella-mediated motility: however, the mechanism for this colonization remains unclear. Here, to obtain clues to this mechanism, we characterized the swimming motility of the Burkholderia symbiont under an advanced optical microscope. High-speed imaging of cells enabled the detection of turn events with up to 5-ms temporal resolution, indicating that cells showed reversal motions $(\theta \sim$ $180^{\circ}$ ) with rapid changes in speed by a factor of 3.6. Remarkably, staining of the flagellar filaments with a fluorescent dye $\mathrm{Cy} 3$ revealed that the flagellar filaments wrap around the cell body with a motion like that of a ribbon streamer in rhythmic gymnastics. A motility assay with total internal reflection fluorescence microscopy revealed that the left-handed flagellum wound around the cell body and propelled it forward by its clockwise rotation. We also detected periodic-fluorescent signals of flagella on the glass surface, suggesting that flagella possibly contacted the solid surface directly and produced a glidinglike motion driven by flagellar rotation. Finally, the wrapping motion was also observed in a symbiotic bacterium of the bobtail squid, Aliivibrio fischeri, suggesting that this motility mode may contribute to migration on the mucus-filled narrow passage connecting to the symbiotic organ.
\end{abstract}

\section{Introduction}

Most swimming bacteria possess a rotary machinery, the flagellar motor, that consists of about 20 different kinds of proteins and is attached to the helical flagellar filament via a hook structure [1]. Despite the structural similarities of

Electronic supplementary material The online version of this article (https://doi.org/10.1038/s41396-017-0010-z) contains supplementary material, which is available to authorized users.

Yoshiaki Kinosita

yoshiaki.kinosita@gmail.com

$\triangle$ Yoshitomo Kikuchi

y-kikuchi@aist.go.jp

1 Department of Physics, Gakushuin University, 1-5-1 Mejiro, Toshima-ku, Tokyo 171-8588, Japan

2 Bioproduction Research Institute, Hokkaido Center, National Institute of Advanced Industrial Science and Technology, Sapporo 062-8517, Japan

3 Present address: Institute for Biology II, Freiburg University, Freiburg, Germany flagella among various species of bacteria, flagellated bacteria show several different swimming patterns during tactic behavior. (i) Cells with peritrichous (randomly arranged on the cell body) flagella (e.g., Escherichia coli and Salmonella enterica serovar Typhimurium) show a linear movement ("run") and sudden reorientation ("tumble") with an angle of $68^{\circ}$ by switching only one or two of the filaments $[2,3]$. (ii) Cells with a single-polar flagellum (e.g., Vibrio alginolyticus) show the forward run-reverse $\left(\Delta \theta \sim 180^{\circ}\right)$-flick $\left(\Delta \theta \sim 90^{\circ}\right)$ behavior by rotating the flagellum in both directions while maintaining its helicity $[4,5]$. (iii) Soil bacteria with peritrichous or lophotrichous flagella (e.g., Sinorhizobium meliloti and Rhizobium lupini) show a forward movement and tumble with an angle of $46^{\circ}$ by alternating the rotation rate of only the right-handed flagellum in three filaments $[6,7]$. When changing their swimming directions, flagellar filaments are often transformed from a normal to curly or semi-coiled state under highly viscous conditions, but their helicity is always right handed. The flagella-mediated motility plays a critical role in the pathogenic cycle, wherein flagellated bacteria form biofilms and/or invade host cells after reaching it by flagellarmediated movement [8]. 
Across a wide range of host-microbe interaction, from pathogenesis to mutualism, flagellar-mediated motility profoundly impacts the outcomes. In the Aliivibrio fischeribobtail squid (Euprymna scolopes) system, A. fischeri first aggregates on ciliated appendages of the squid light organ [9]. Although the flagella-mediated motility is not required at this stage, it is essential for migrating through pores and into the ducts to colonize on internal crypts of the host cells. In the Burkholderia sp. RPE64-Riptortus pedestris system, the bean bug acquires Burkholderia cells from the ambient environment and exclusively possesses the symbiont in the lumen of the midgut crypts [10]. To select the symbiont from environmental contaminants, this insect has developed a sophisticated symbiont-sorting apparatus in the gut: it constricts the anterior part of the crypt-bearing region of the midgut, which contains a narrow passage filled with a polysaccharide-rich mucous matrix. Until recently the mechanism for the selective incorporation had been unclear, but a recent study demonstrated that flagella-mediated motility of the Burkholderia symbiont is pivotal for the transfer through the narrow passage of the constricted region [11]. Notably, nonsymbiotic motile bacteria such as E. coli and Bacillus subtilis are unable to pass through the constricted region, suggesting that Burkholderia has a distinct motility mode from these flagellated bacteria. To test this hypothesis, we stained flagellar filaments of Burkholderia sp. RPE64 with a fluorescent dye and directly visualized them under a fluorescent microscope. By using a total internal reflection fluorescence microscope (TIRFM), we demonstrated the surface movement driven by flagellar rotation on a solid surface.

\section{Materials and methods}

\section{Strain and cultivation}

Burkholderia sp. RPE64 cells were grown in YG medium $(0.5 \%$ (wt/vol) yeast extract, $0.4 \%$ (wt/vol) glucose, $0.1 \%$ $\mathrm{NaCl}$ ) at $25^{\circ} \mathrm{C}$ with shaking to an optical density of around 0.7 at $600 \mathrm{~nm}$. We also used two motility mutants with Tn5insertions in their flagella-related genes: AM3, a mutant with a Tn5-insertion into a chemotaxis-related gene (cheA), and NM7, a non-motility mutant with a Tn5-isertion into its motor-switch gene (fliM) [11].

\section{Preparation of the fluorescent-labeled cell}

Burkholderia symbiont cells in $1 \mathrm{ml}$ of medium were collected by centrifugation at $5000 \times g$ for $4 \mathrm{~min}$ at $25^{\circ} \mathrm{C}$, resuspended in $1 \mathrm{ml}$ of $0.1 \mathrm{M}$ sodium phosphate buffer pH 7.8 containing Cy3-NHS-ester (GE Healthcare), and incubated for $10 \mathrm{~min}$ at room temperature [2, 12]. After labeling, the excess fluorescent dye was removed by two rounds of washing, and fluorescent-labeled cells were resuspended in YG medium.

\section{Motility assay}

All experiments were performed at room temperature. The flow chamber was comprised of two coverslips (see Supplementary Methods). In the swimming assay, the flow chamber was first infused with medium containing $5 \mathrm{mg} / \mathrm{ml}$ bovine serum albumin (Sigma Aldrich), and then with $10 \mu \mathrm{l}$ of the cell-suspension medium. For observation of stuck cells on a glass surface under a total internal reflection fluorescence microscope, cells were directly added into the chamber $(18 \times 18$ and $22 \times 32 \mathrm{~mm})$ without glowdischarge.

Viscous agents, including Ficoll (F4375; Sigma Aldrich) and methylcellulose (M0512; Sigma Aldrich), were infused into the flow chamber containing the cells.

\section{Microscopy}

To observe swimming cells, cells were visualized by a mercury lamp under an inverted microscope (IX71; Olympus) equipped with a $\times 40$ objective (UPLFLN-PH, NA 0.75, Olympus), a CCD camera (HDR1540H, Digimo), a highly stable customized stage (Chukousha) and an optical table (RS-2000, Newport).

For visualization of fluorescent-labeled cells, a green laser beam (Compass-315M-100, wavelength of $532 \mathrm{~nm}$; Coherent) was introduced into an inverted microscope equipped with a $\times 100$ objective (Plan Apo TIRF, NA 1.49; Nikon Instruments), a dichroic mirror (custom-made; Chroma), an emission filter (NF01-532U; Semrock), and an EMCCD camera (iXon ${ }^{+}$DU860; Andor).

\section{Data analysis}

Reorientation events were identified as previously described [4]. Fluorescent micrographs were recorded at $2.5 \mathrm{~ms}-$ intervals and captured as 16-bit images, using an EMCCD camera with a resolution of $130 \times 130 \mathrm{~nm}^{2}$ per pixel on the camera plate. The swimming speed and rotation rate of fluorescent-labeled cells were measured by making a kymograph [13]. Under TIRF illumination, the rotation rate was calculated by fast Fourier transform analysis (see Supplementary Methods). Integrated images were constructed using ImageJ $1.48 \mathrm{v}$ software (http://rsb.info.nih.gov/ij/).

Full methods are described in the Supplementary Information. 
a

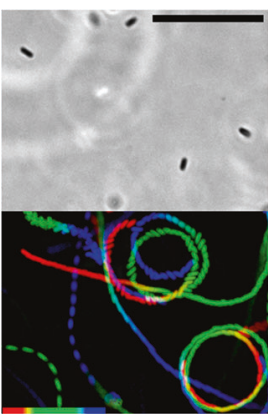

d

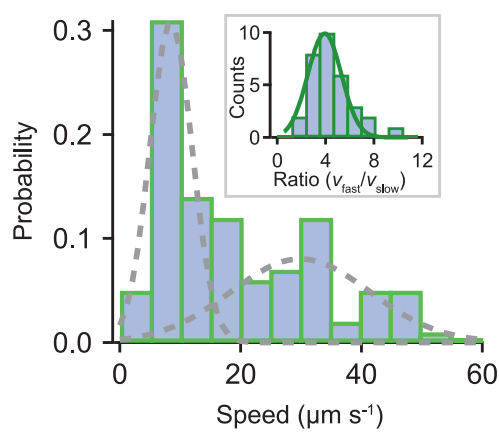

b

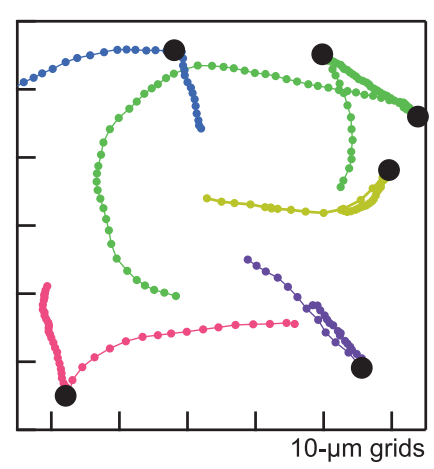

C

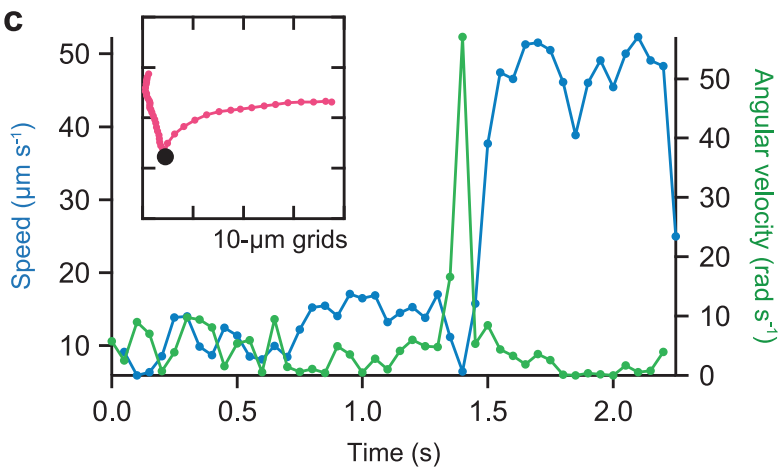

f

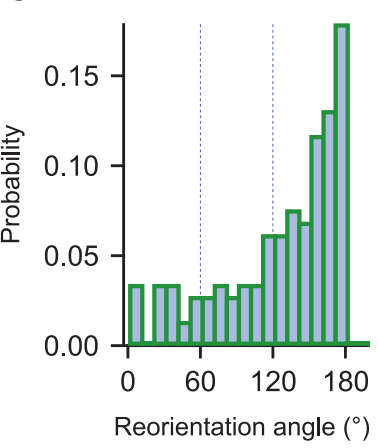

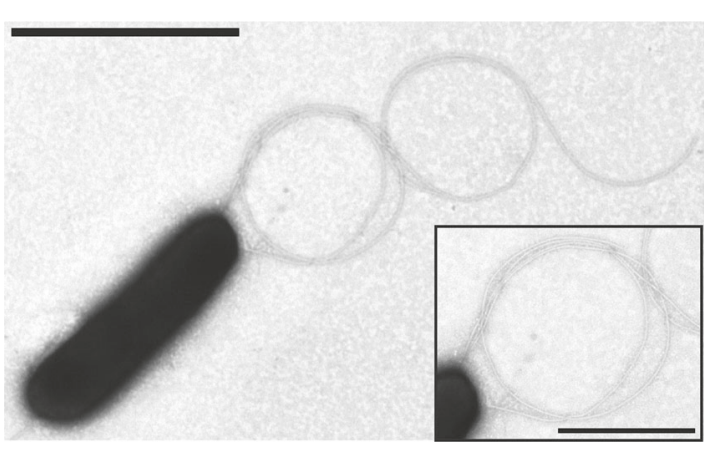

Fig. 1 Characterization of a swimming motility and structural parameters of a symbiotic bacterium, Burkholderia sp. RPE64. a Upper: phase-contrast image of Burkholderia sp. RPE64. Scale bar, $20 \mu \mathrm{m}$. Bottom: sequential phase-contrast images taken at $50 \mathrm{~ms}$ - intervals over a period of $5 \mathrm{~s}$ were integrated with the intermittent color code "red $\rightarrow$ yellow $\rightarrow$ green $\rightarrow$ cyan $\rightarrow$ blue". b Typical example of swimming trajectories with a turn events (black dots). Each dot represents one of the images at point with $50 \mathrm{~ms}$-intervals. Different colors denote the trajectories of different cells. c Time course of the swimming speed $(v$, cyan) and angular velocity ( $\omega$, green). Inset: $x-y$ trace with a reversal motion. d The probability of different swimming speeds for cells. The dotted line represents a Gaussian distribution, where the peaks and SDs were $8.5 \pm 3.5$ and $29.9 \pm 11.5 \mu \mathrm{m} \mathrm{s}^{-1}(n=100)$. Inset: histogram of the ratio between $v_{\text {fast }}$ and $v_{\text {slow. }}$ The peak and SD was $3.9 \pm 1.4(n=32)$. e Turning angle distribution of swimming Burkholderia symbiont cells. The total number of analyzed turns is 145 in 73 cells. f Electron micrograph of the cell body. Scale bars, $2 \mu \mathrm{m}$. Inset: the magnified image of coiled-flagellar filaments. Scale bar, $1 \mu \mathrm{m}$

\section{Results}

\section{Swimming motility and flagellar structure of Burkholderia sp. RPE64}

To better understand the motility mechanism of the Burkholderia symbiont, we first investigated the swimming speed and motility pattern under a phase-contrast microscope (Supplementary Movie 1, Fig. 1a upper). Most trajectories were either linear or orbital (Fig. 1a bottom), and reversal motions were also detected as seen in polarflagellated bacteria [4,5] (Fig. 1b). When the cells changed their swimming direction, the swimming speed rapidly changed (Fig. 1c). To evaluate the difference in swimming speed after the turn, we made a histogram of the swimming speed for each cell (Supplementary Fig. 1). The speed distribution of cells had two peaks, where the $v_{\text {slow }}$ and $v_{\text {fast }}$ peaks exhibited a speed of $8.5 \pm 3.5$ and $29.9 \pm 11.5 \mu \mathrm{m} \mathrm{s}^{-1}$ $(n=100$, Fig. 1d). We next analyzed cells with reversal events, the ratio of swimming speed $\left(v_{\text {fast }} / v_{\text {slow }}\right)$ in each cell was estimated to be $3.9 \pm 1.4$ (Fig. 1d inset). To characterize the switching behaviors in detail, we next extracted angle changes between $\theta(t)$ and $\theta(t+\Delta t)$ from the algorithm based on [4] (see Supplementary Methods). For the turning angle, we found that the maximum of angle changes was $\sim 180^{\circ}$, that is, the cells reversed their direction of motion (Fig. 1e). This motility pattern in which cells reverse their direction with a speed change has been previously observed in soil bacterial species, Pseudomonas putida and Pseudomonas fluorescence [14, 15].

Next, we analyzed the flagellar morphology under transmission electron microscopy (TEM) (Fig. 1f). The flagellar filament was located at a cell pole, and the average number of filaments was approximately 3 with a length of $5.6 \pm 1.1 \mu \mathrm{m}(n=25)$. The flagellar pitch and helix radius were estimated to be $2.6 \pm 0.5 \mu \mathrm{m}$ and $0.26 \pm 0.07 \mu \mathrm{m}$, respectively, which were similar to the values for the normal state in S. typhimurium flagella [16]. In addition, we frequently observed filaments to form circular shapes with radius of $0.63 \mu \mathrm{m}$, which have been called coiled-state 
a

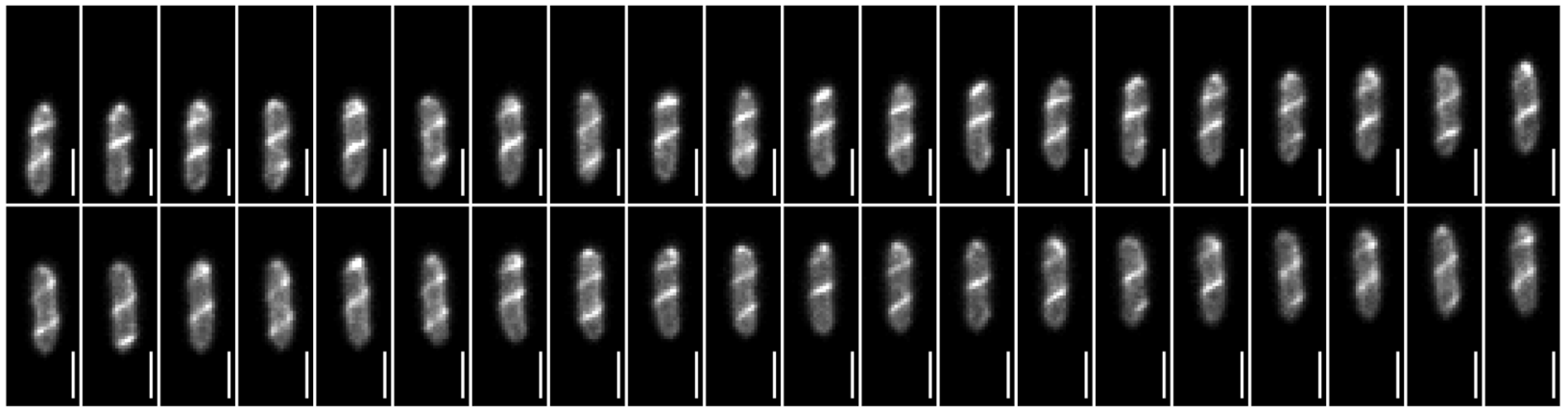

b

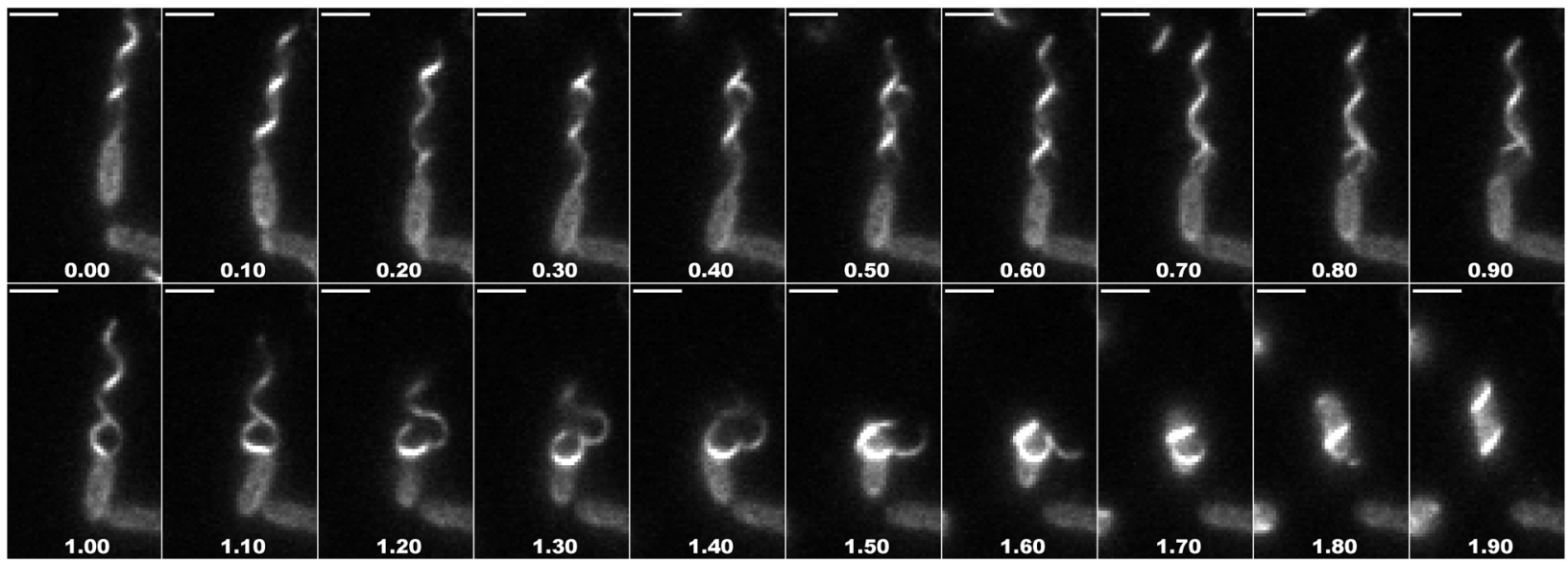

Fig. 2 Wrapping motion of Burkholderia sp. RPE64. a Sequential fluorescent images of a wrapping motion in a free-swimming state at 25 ms-intervals for $1 \mathrm{~s}$. The tip of the flagella (hook) was located at the top of the cell body. The flagellar wave propagated in a direction from the top end to bottom end of the cell body. Scale bar, $2 \mu \mathrm{m}$. b Change of the swimming mode from the normal to wrapping motion at 100

filaments in other flagellated bacteria (Fig. If inset, Supplementary Results 1) [16]. Other structural parameters are summarized in Supplementary Table 1.

\section{Visualization of a wrapping motion under fluorescent microscopy}

To directly visualize flagellar dynamics of the Burkholderia symbiont, we stained the flagellar filaments with a fluorescent dye, Cy3-NHS-ester (see Material and Methods section). To track the swimming motion of labeled cells, we used an electron-multiplying charged-coupled device (EMCCD) camera, which enabled detection of the flagellar dynamics with a high $\mathrm{S} / \mathrm{N}$ ratio and with a time resolution of $2.5 \mathrm{~ms}$. Remarkably, we found two swimming modes in this assay: (i) a motion in which the flagella bundles push the cell body (hereinafter the normal motion; Supplementary Movie 2); (ii) a motion in which the flagella wind around the cell body with a motion like that of a ribbon streamer in rhythmic gymnastics (Fig. 2a and Supplementary Movie 3). ms-intervals. From 0 to $0.3 \mathrm{~s}$, the wave of flagella propagates away from the cell body. From 0.4 to $0.9 \mathrm{~s}$, the rotation of flagella stopped. From 1 to $1.4 \mathrm{~s}$, the normal-shaped flagella gradually changed to a coiled state, then wound around the cell body at from 1.5 to $1.9 \mathrm{~s}$. The flagellar wave was propagated in a direction from the flagella end to the cell body. Scale bar, $2 \mu \mathrm{m}$

Additionally, we frequently observed changes in the swimming mode from the normal to the wrapping motion and vice versa (Fig. 2b, Supplementary Movie 4).

To explore differences between the two swimming modes, the swimming speed and rotational rate of flagella were characterized by performing a kymograph analysis (Supplementary Fig. 2). In the normal motion, the swimming speed and the rotational rate were $23.7 \pm 7.5 \mu \mathrm{m} \mathrm{s}^{-1}$ and $154.0 \pm 41.8 \mathrm{~Hz}(n=41)$, respectively. In contrast, these parameters were $7.6 \pm 4.6 \mu \mathrm{m} \mathrm{s}^{-1}$ and $52.9 \pm 14.3 \mathrm{~Hz}$ $(n=88)$ in the wrapping motion, or approximately three times smaller than in the normal motion. The rate of reduction in the swimming speed was consistent with the observation under the phase-contrast microscope, indicating that this wrapping motion was a natural phenomenon, and not due to the fluorescent labeling.

We next examined the effect of viscosity on the swimming speed and rotational rate of flagella. Although the viscosity of the gut ducts in the bean bug remains under investigation due to the difficulty of the collection, the 
a

Twining flagella around the cell body

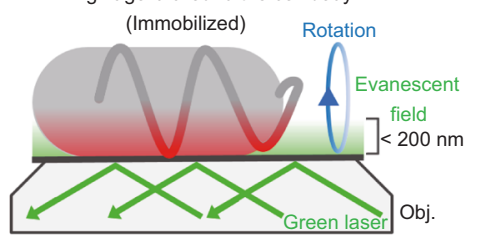

b

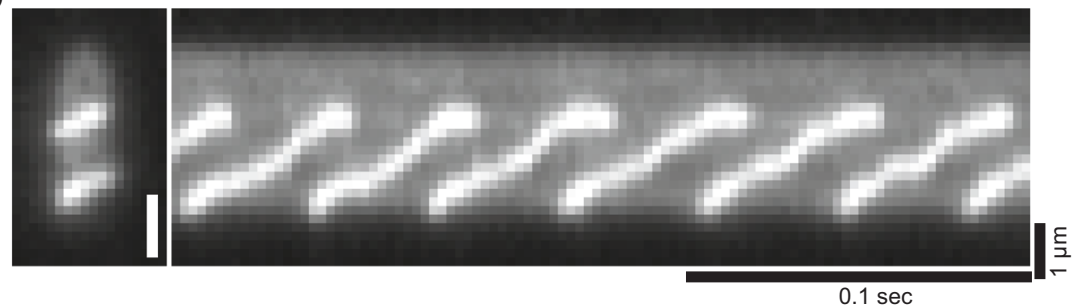

d
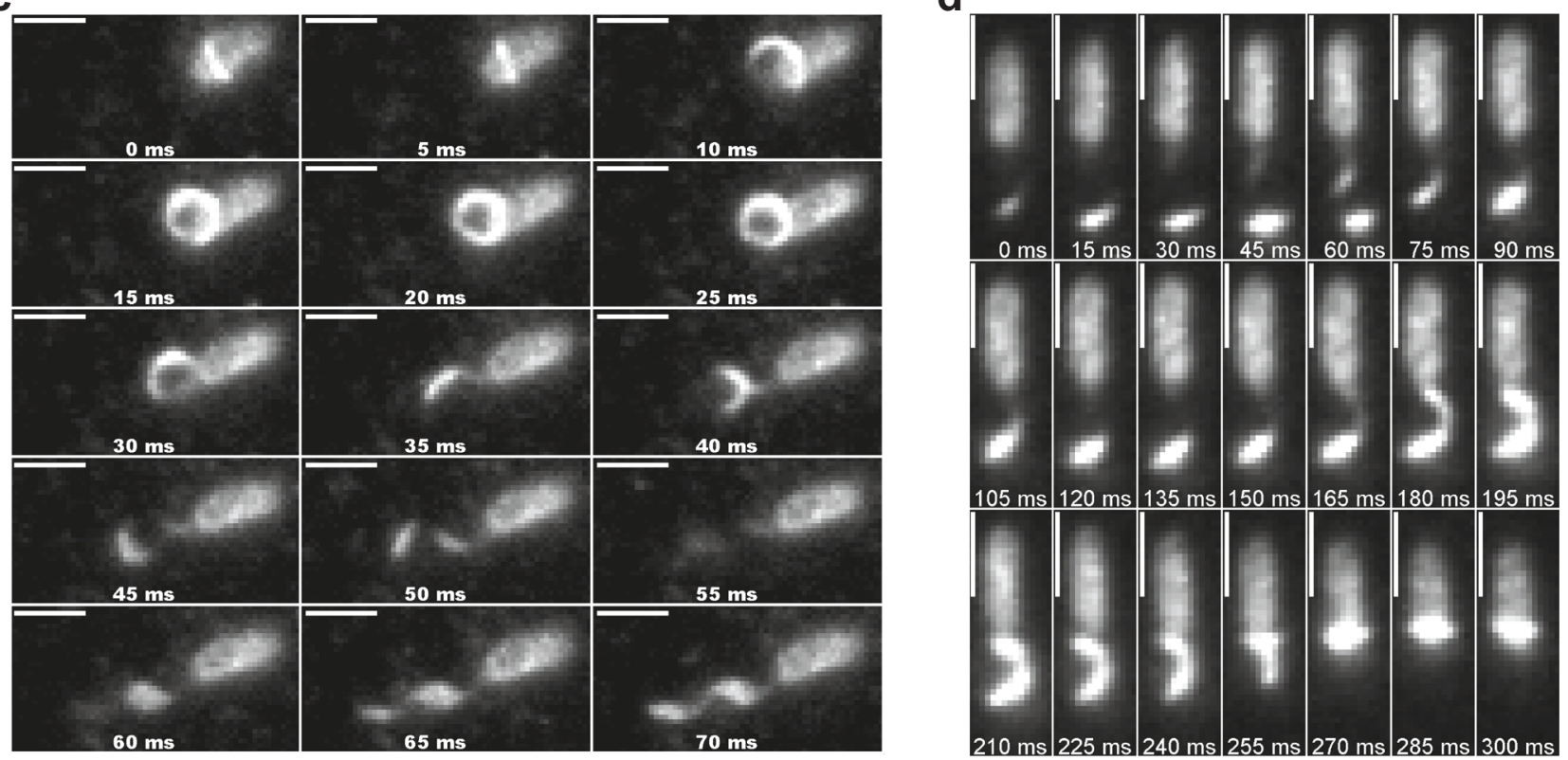

e

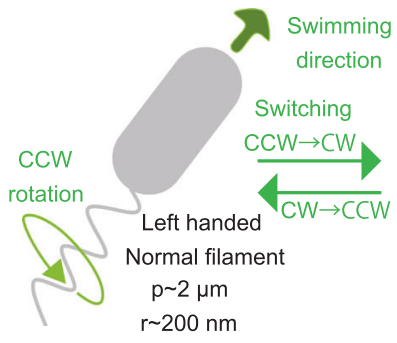

Fig. 3 Determination of helicity and rotational direction of flagella during the wrapping motion under TIRFM. a Schematic of the experimental setup. b Left: an image of a single cell during the wrapping mode. Flagella were located on the bottom side. As shown in the picture, the orientation of flagella was from the 1st quadrant to the 3rd quadrant relative to the major axis of the cell body, indicating that their helicities were left-handed. Note that because our observation was carried out using an inverted microscope, all images we presented in this article are mirror images. Scale bar, $1 \mu \mathrm{m}$. Right: kymograph of the left image for $0.25 \mathrm{~s}$. The wave of flagella propagated in a direction away from the flagella end and toward the cell body, indicating that

mucus of animals were estimated to be in the range of $1-1000 \mathrm{cP}$ [17]. To replicate different natural conditions, we used two kinds of viscous agents: (i) methylcellulose, an unbranched polymer that is non-newtonian at concentrations over $0.5 \%$; (ii) Ficoll, a newtonian and highly branched polymer. From the product information and previous

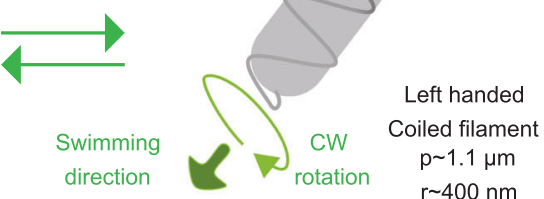

rotated in a $\mathrm{CW}$ rotation. c Sequential fluorescent images of the flagellar polymorphism at $5 \mathrm{~ms}$-intervals. At $10 \mathrm{~ms}$, the flagella were unwound from the cell body. Coiled flagella were twisted at $30 \mathrm{~ms}$, and then their form changed from a coiled to a normal state at $60 \mathrm{~ms}$. After the polymorphic transformation, the wave of flagella propagated in a direction away from the hook end toward the flagellar tip, indicating that the flagella rotated in a CCW rotation. Scale bar, $2 \mu \mathrm{m}$. d Sequential fluorescent images of the flagellar transformation from a normal to a coiled form at $15 \mathrm{~ms}$-intervals. Scale bar, $2 \mu \mathrm{m}$. e Schematic of a swimming model of Burkholderia sp. RPE64 based on the above observation. $\mathrm{p}$ pitch of flagella, $r$ radius of flagella

report [18], the viscosities of $15 \%$ (wt/vol) Ficoll, 0.3\%, $0.5 \%$, and $0.8 \%$ (wt/vol) methylcellulose were estimated to be $10,13,26$ and $71 \mathrm{cps}$ at R.T., respectively.

We observed the wrapping motion regardless of the viscous agent (Supplementary Fig. 2). In the presence of $0.3 \%$ methylcellulose, the swimming speed/rotational rate 
during the normal and wrapping motion were $13.4 \pm 5.3$ $\mu \mathrm{m} \mathrm{s}^{-1} / 58.9 \pm 17.9 \mathrm{~Hz}(n=34)$ and $8.4 \pm 3.7 \mu \mathrm{m} \mathrm{s}^{-1} / 36.1$ $\pm 11.8 \mathrm{~Hz}(n=64)$, respectively. Under the same viscous conditions established using Ficoll, the swimming speed/ rotational rate during the normal and wrapping motion were $20.2 \pm 10.3 \mu \mathrm{m} \mathrm{s}^{-1} / 125.9 \pm 40.7 \mathrm{~Hz}(n=37)$ and $5.0 \pm 3.2$ $\mu \mathrm{m} \mathrm{s}^{-1} / 40.3 \pm 12.7 \mathrm{~Hz}(n=45)$, respectively. Despite the similar viscosities, the flagellar rotation of wrapping cells was two times more efficient ( $v$ swimming speed/ f rotation rate) in methylcellulose compared to Ficoll. This suggests that the cell body, which fluctuates and/or rotates relative to the swimming axis, was hindered by the gel-like structure consisting of unbranched polymers. In gels-like structures consisting of unbranched polymers the flagella could push effectively without slipping $[19,20]$. The results under other viscous conditions are described in Supplementary Fig. 2.

\section{Determination of the helicity and rotational direction of the flagellar filament}

Although we detected the flagellar dynamics and polymorphism under both swimming modes, the helicities were not determined under epifluorescence. This is because we could not judge which side, the top or the bottom, of curved flagella was in the focus plane in free-swimming. To solve this problem, we constructed a flagella observation system using a total internal reflection fluorescence microscope TIRFM (Fig. 3a). This system enables the detection of fluorescent signals at $<200 \mathrm{~nm}$ depth from the glass surface. Therefore, the helicity of flagella can be determined by the orientation of the near-surface segment of a filament (for details see Ref. [13]).

First, we wanted to establish a set of conditions under which the cell body would attach to the glass surface. In the absence of viscous agent, a few percent of cells were stuck on the glass surface and showed both normal and wrapping motion. In both cases, the portion of flagella that made contacts with the evanescent field was visible as short lines aligned northeast-southwest relative to the major axis of the cell body aligning north-south as a mirror image under the inverted microscope, indicating the left-handed helicity (Fig. 3b left, Supplementary Movie 5). The left-handed flagella wave propagated away in the normal mode and towards the cell body in the wrapping mode, which guaranteed the motor rotated in the counterclockwise (CCW) and clockwise (CW) direction, respectively (Fig. 3b right, Supplementary Movie 5). The flagellar morphology and rotation rate in both modes are shown in Supplementary Results 2.

The flagellar polymorphism of stuck cells on a glass surface was often observed under TIRF illumination (Supplementary Movie 6). When wrapping flagella unwind from a cell body, the coiled-shaped flagella are observed
(Fig. 3c). At $35 \mathrm{~ms}$ in Fig. 3c, the orientation of the flagellar filament is northwest with respect to the long axis of cell body, indicating that the flagellar-filament is right-handed. At $50 \mathrm{~ms}$, we observed the northwest- and northeast-shaped flagellar filament, and found that the left- and right-handed flagellar filaments were combined into a single filament, which was also observed in a flagellar filament of $E$. coli [21]. At $60 \mathrm{~ms}$, the left-handed normal filament was visualized. These results suggested that the flagellar filament changed not only its helical pitch and radius but also its handedness during the switch.

\section{Cell body rotation}

We also observed free-swimming cells to check the rotational direction of the cell body under TIRF illuminations. Cell bodies were visible when the cells swam in the vicinity of the glass. In the presence of $0.5 \%$ methylcellulose, most cells did not stick on the glass surface and freely swam in the medium (Supplementary Movie 7). Note that there were only sparse fluorescent signals from the cell body in this assay; therefore, the rotational direction of the cell body was determined based on the direction of the spot's movement. For analyses, we defined the observation configuration with flagella aligned parallel to the north-south orientation and the cell body located at the north. If the sparse pattern moved from the east to the west, this indicated that the cell body rotated in the $\mathrm{CW}$ direction. From this analysis, the directions of cell body rotation during the normal and wrapping modes were determined to be $\mathrm{CW}$ and $\mathrm{CCW}$, respectively. Because flagellar filaments rotate in the $\mathrm{CCW}$ and $\mathrm{CW}$ directions, this result indicated that the cell body counter-rotated against the flagella rotation to balance the torque. Additionally, we also observed the flagellar polymorphism in this assay (Fig. 3d). When the normal mode changed to the wrapping mode, the rotational direction changed from the $\mathrm{CCW}$ to the $\mathrm{CW}$ direction. This switching triggered the flagellar polymorphic transition from the normal state to the coiled state. At $180 \mathrm{~ms}$ in Fig. 3d, we observed the right- and left handed flagellar filament, which subsequently wound around their cell body. Based on the above observations, the rotational direction, helicity and polymorphism of flagella can be schematically summarized in Fig. 3e and described as follows: (i) the left-handed flagellar filament rotates in the $\mathrm{CCW}$ direction; (ii) the flagellar rotation stops; (iii) the left-handed normal filaments are transformed into the right-handed and/or left-handedcoiled form when the motor rotates in the $\mathrm{CW}$ direction; (iv) the right-handed flagellar filaments instantaneously wind around their cell body and are transformed to left-handed flagella; and (v) the CW rotation of the left-handed flagellar filaments propels the cell in the opposite direction of the normal motion. 
a
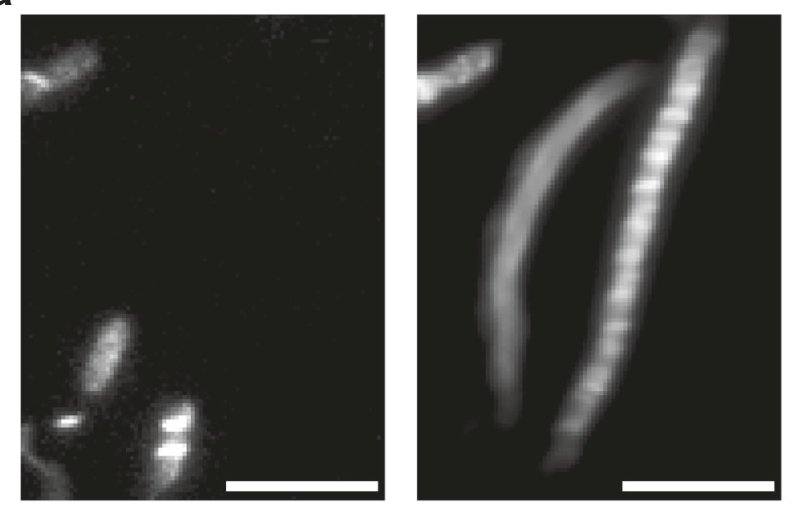

C

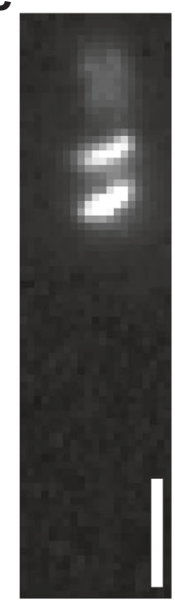

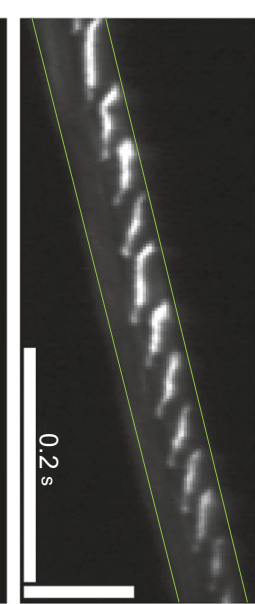

d

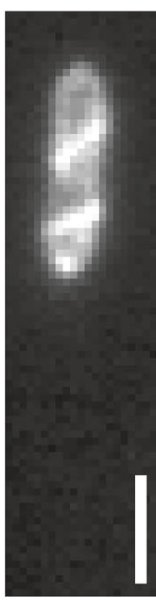

b
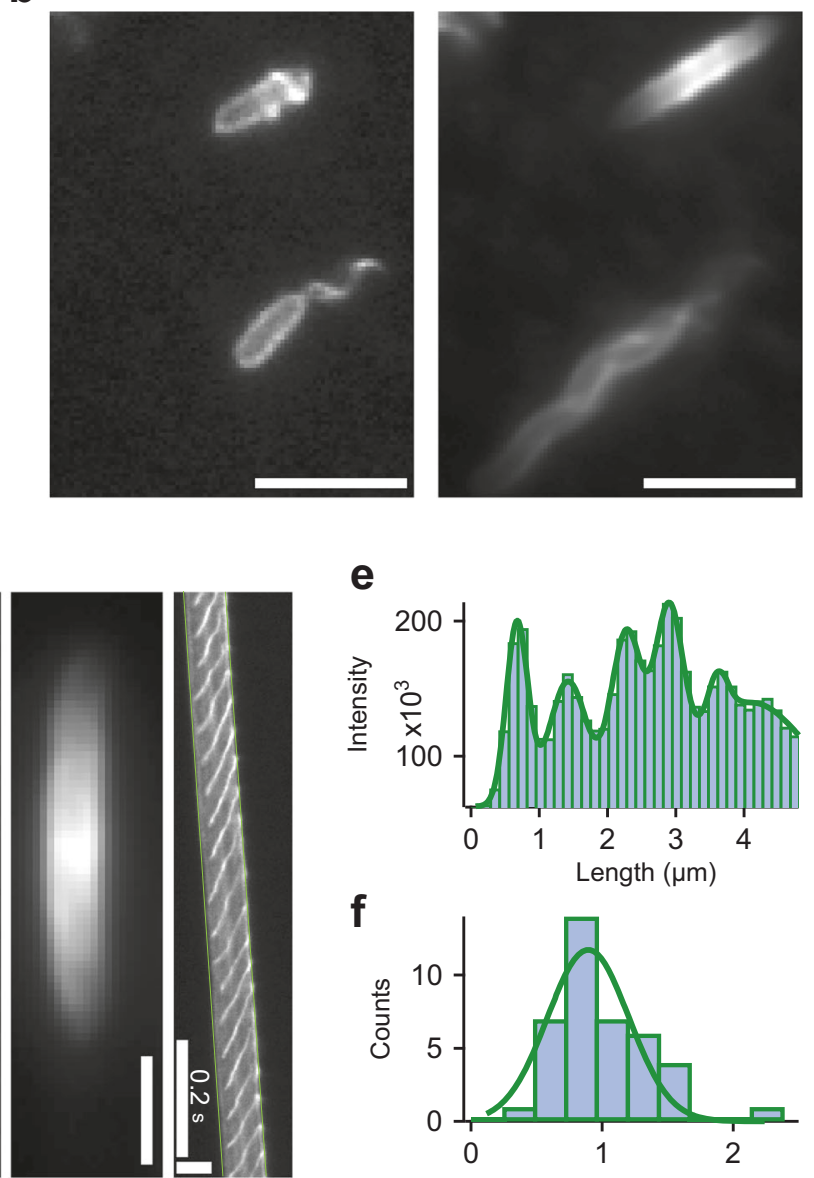

e

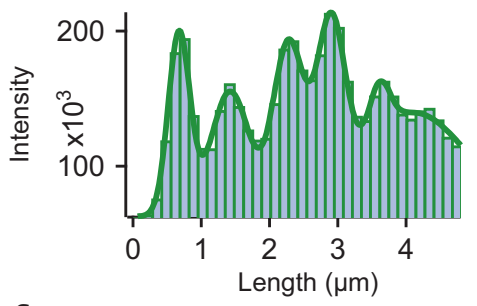

f

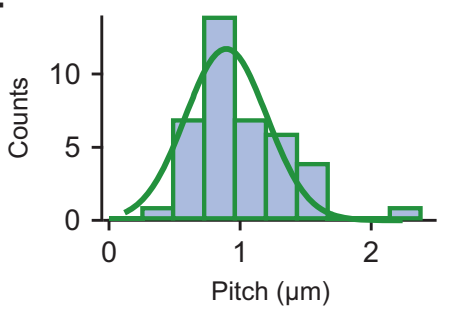

Fig. 4 Wrapping motion produce a driving force on a solid surface. a A still image of the swimming motility near a glass surface (left) and the integrated image of the image at left for $1 \mathrm{~s}$ (right) obtained by TIRFM. Scale bar, $5 \mu \mathrm{m}$. b A still image of the swimming motility of Burkholderia sp. RPE64 in the solution (left) and an integrated image of the image at left for $1 \mathrm{~s}$ (right) under epifluorescence illumination. Scale bar, $5 \mu \mathrm{m}$. Still images obtained under TIRFM $\mathbf{c}$ and an epifluorescent microscope d. Left: A still image. Middle: The sum-type of

\section{Gliding-like motility driven by the wrapping motion}

Unexpectedly, the cells in the wrapping mode frequently move near the glass surface and showed unidirectional displacement without stuck. This successive movement could never have been observed in $E$. coli, once E. coli was tethered on the glass surface (Supplementary Movie 8). To gain insights into this surface movement in the Burkholderia symbiont, we made an integrated image of the sequential-fluorescent images over 1-2.5 s, which enabled the direct detection of the interaction between the solid surface and flagella [22]. Notably, the periodic patterns were frequently detected in wrapping cells near the glass surface under TIRF illumination ( $41 \%$ in $n=90$; e.g., Fig. $4 a, c)$. In contrast, we could not observe any pattern for projection. Right: The kymograph of a middle part of the left image. The green line represents the location of the cell body. Scale bar, $2 \mu \mathrm{m}$ (left) and $3 \mu \mathrm{m}$ (right). e An example of the intensity profile of the periodic change of wrapping motion along the green line in the middle panel of c. f Histogram of the length between fluorescent signals as shown in e, motile pitch $(n=40)$. The green line represents the Gaussian fitting. The peak and s.d. were $0.89 \pm 0.31 \mu \mathrm{m}$

free-swimming cells under epifluorescence illumination (Fig. 4b, d). To further investigate the difference between a wrapping cell in solution and a wrapping cell near the glass surface, we performed a kymograph analysis (Fig. 4c right). In this analysis we observed two different phenomena: (i) when a cell moved near the glass surface, the flagellar propagation suddenly slowed down and was transiently moved in the direction of the tip (Fig. 4c); (ii) when cells swam in solution, the flagellar filaments propagated in the opposite direction of the cellular swimming direction (Fig. 4d). Notably, phenomenon (i) could not be detected for wrapping cells in solution (Fig. $4 \mathrm{~d}$ right). It is possible that during the described phenomenon (i), the flagellar filament interacts transiently with the surface and subsequently interacts along the same path traces the same 
position relative to the surface, and thus the periodic pattern in the integrated like Fig. $4 \mathrm{c}$ right emerges with the interaction. This observation suggested that the interaction between the flagellar helix and surface was essential to achieve successive movement on surface while preventing cells to get stuck (see Discussion section for more details).

We next drew a straight line along the fluorescent patterns and quantified the periodicity between signals (Fig. 4c middle, e). The motile pitch was calculated to be $0.89 \pm$ $0.31 \mu \mathrm{m}$ ( $n=40$, Fig. $4 \mathrm{f})$, which was similar to the structural pitch of the flagella of an immobilized cell (Supplementary Fig. 3b).

\section{Comparison of motilities between the wild type and mutant}

We next conducted a mutant analysis to further clarify the mechanism by which the flagella wrap around the cell body. In the wild type (WT) observation, we found that the direction of flagellar rotation switched upon flagellar transformation from the normal to coiled form and vice versa (Fig. 3). To analyze the effect of switching rotation on the flagellar transformation, we analyzed the motility of the cheA_AM3 mutant [11]. In this mutant CheA is constantly inactive, resulting in dysfunction of the response regulator CheY. As disruption of the CheY binding to the FliM protein in the flagellar switch complex, the cheA_AM3 mutant is locked in CCW rotation [1]. We expected that this mutant only showed the normal motion, and not the wrapping motion. The structural parameters of flagellar filament were shown in Supplementary Table 1.

To address whether the wrapping motion was inhibited in the chemotaxis mutant, we observed its flagellar dynamics under a fluorescent microscope (Supplementary Movie 9). As expected, we observed only normal motion during the swimming motility of AM3, and its swimming speed and flagellar rotation rate were estimated to be $10.2 \pm 3.7 \mu \mathrm{ms}^{-1}$ and $92.9 \pm 32.6 \mathrm{~Hz}$ by taking a kymograph, respectively ( $n=38$; Supplementary Fig. 4). Somewhat surprisingly, although the swimming speed of AM3 was half that of WT, the colony diameter of AM3 was 8 times smaller than that of WT (Supplementary Fig. 4c). It suggested that the switching behavior was essential for ring formation in semi-solid agar plate [23]. Besides, we speculate that the wrapping motion is useful for migration on the solid surface, taking into account that the mechanical contact is important for migration on such surface [24].

The coil-shaped flagellum was infrequently observed under a fluorescent microscope (Supplementary Fig. 4d). The effect of a motility on the flagella transformation is shown in Supplementary Results 3.
Fig. 5 Detection of a wrapping motion in another symbiotic bacterium, Aliivibrio fischeri. a Electron micrograph of the cell body. Scale bar, $2 \mu \mathrm{m}$. b Typical example of swimming

trajectories with a turn event (black dots). Each dot represents one of the points at $50-\mathrm{ms}$ intervals. Different colors denote trajectories of different cells. c Turning angle distribution of swimming A. fischeri cells. The total number of analyzed reversals is 121 in 60 cells. d The probability of different swimming speeds of cells $(\mathrm{n}=$ 97). The solid lines represent the sum of two Gaussians, where peaks and SDs were $14.1 \pm 3.7$, $49.4 \pm 14.8 \mu \mathrm{m} \mathrm{s}^{-1}$. Inset: Histogram of the ratio between $v_{\text {fast }}$ and $v_{\text {slow }}$. The peak and SD was $3.4 \pm 0.6(n=43)$. e

Fluorescent micrograph of a cell labeled by a membrane-specific dye and amine-specific dye, FM4-64 and Cy3-NHS-ester, respectively. The normal (upper) and wrapping mode (lower) are shown. Scale bar, $2 \mu \mathrm{m}$ a
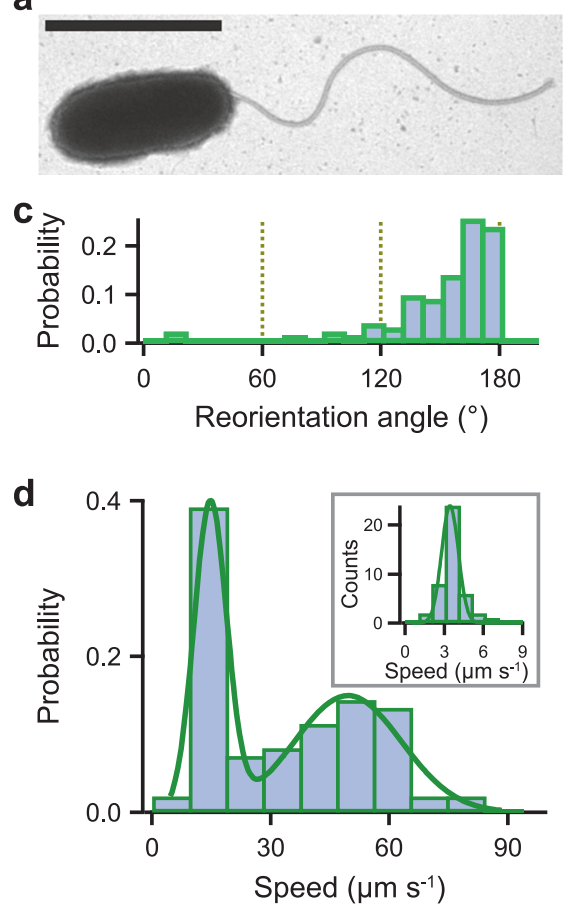

b

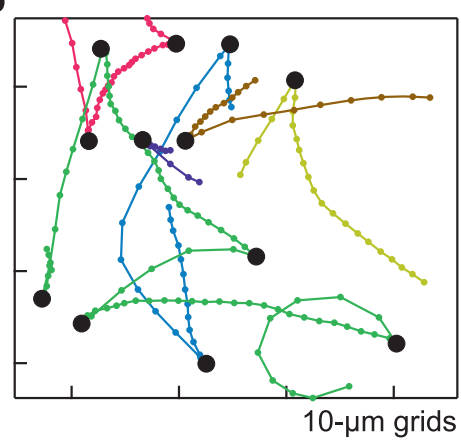

e

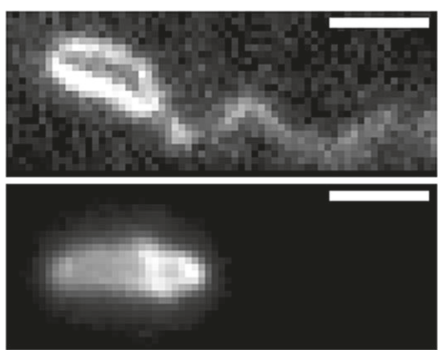




\section{Detection of wrapping motion in a symbiotic bacterium, Aliivibrio fischeri}

To investigate whether the wrapping motion was a feature inherent to the Burkholderia symbiont, we attempted to detect the flagellar dynamics of another symbiotic bacterium, Aliivibrio fischeri. A. fischeri, which is found in marine animals such as the bobtail squid, possesses between one and three polar flagella and exhibits a swimming motility, which is essential for its colonization of host-cell crypts [9, 25]. We first checked the flagellar morphologies of this species under TEM (Fig. 5a). As previously reported, $A$. fischeri formed a few flagella at the cell pole, and the average number of flagella was approximately one $(1.3 \pm$ $0.7 ; n=39$ ). Other structural parameters, such as the flagellar pitch and helix radius, are described in Supplementary Table 1.

Next, using the same approach as for the Burkholderia symbiont, we analyzed the swimming speed and mode of $A$. fischeri under a phase-contrast microscope (Supplementary Movie 10). Most cells showed straight motion and rapidly changed their swimming direction with an average angle of $\sim 180^{\circ} \quad(n=121$, Fig. 5b, c). Interestingly, A. fischeri exhibited a feature similar to that of the Burkholderia symbiont: it reduced its swimming speed by more than half after changing its swimming direction (Supplementary Fig. 6). The average swimming speeds of $v_{\text {fast }}$ and $v_{\text {slow }}$ were $49.4 \pm 14.8$ and $14.1 \pm 3.7 \mu \mathrm{m} \mathrm{s}^{-1} \quad(n=97$, Fig. 5d $)$ respectively, and the ratio of swimming speed $\left(v_{\text {fast }} / v_{\text {slow }}\right)$ in each cell was $3.4 \pm 0.6$ ( $n=43$, Fig. $5 \mathrm{~d}$ inset). This value was consistent with that of the Burkholderia symbiont, suggesting that $A$. fischeri had a similar switching manner of switching its pattern of motion to approach preferred environments and/or establish symbiotic relationship with its host.

To directly determine whether A. fischeri showed a wrapping motion, we here developed a method to label filaments of A. fischeri with a membrane-specific dye, FM464 (see the Supplementary Methods). Although the fluorescent signal was slightly low, a flagellar dynamics of up to

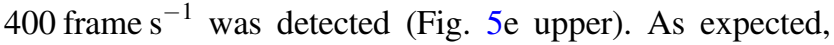
we detected the wrapping motion in A. fischeri; we also detected switching behaviors from a normal to wrapping motion and vice versa, which were triggered by a change in the rotational directions of the flagellar filaments (Fig. 5e lower and Supplementary Movie 11).

\section{Discussion}

In this study, we demonstrated the wrapping motion of the symbiotic bacteria Burkholderia sp. RPE64 and Aliivibrio fischeri under a fluorescent microscope. Previous studies showed that the Burkholderia symbiont passes through a midgut-constricted region filled with a polysaccharide-rich mucous matrix which non-symbiotic bacteria such as $E$. coli and Bacillus subtilis are unable to pass through [11]. In addition, $A$. fischeri cells selectively aggregate and migrate thorough the pores and into the ducts to colonize on the internal crypts of host cells [9]. A similar narrow entrance to the symbiotic organ has been reported in other animals, such as earthworms. Verminephrobacter symbionts in the earth worm also pass through a narrow entrance known as a "colonization duct" during host embryogenesis [26]. Notably, flagella motility is also pivotal for the symbiont colonization in the earthworm system [27]. We highly expect that an unforeseen motile mechanism will be found to play a pivotal role in these symbiotic associations, possibly revealed by an advanced microscopic technology such as a TIRFM.

The symbiotic bacteria also develop other colonization factors in addition to their unique wrapping behavior. For example, a diverse range of colonization factors, such as cell wall synthesis [28], lipopolysaccharide synthesis [29, 30], purine synthesis [31], and polyester synthesis [32], have been reported in the Burkholderia symbiont. In A. fischeri, a number of colonization factors (reviewed in [9, 33] including quorum-sensing [34] and tolerance of oxidative stress $[35,36]$ have been identified. We infer that the novel wrapping motion is necessary to ensure specific colonization in the symbiotic organ in addition to these colonization factor.

We also observed the flagellar-driven gliding-like motion in the Burkholderia symbiont (Fig. 4). We found the periodical fluorescent signals of the wrapping motion near the glass surface, of which the periodicity between signals was consistent with the flagellar helical pitch (Fig. 4a, f). This result suggests that the flagellar filament temporarily maintains the same position relative to the surface, and its position changes with time as a consequence of the continuous flagellar rotation around its cell body. In addition, in these cells near the glass surface, the flagellar wave propagation suddenly slowed down and moved to the opposite direction of the flagellar end as shown by a kymograph analysis (Fig. 4c right). This suggests that the flagellar helix instantaneously interacts with the glass surface and afterwards transiently changed the interaction point by continuous flagellar rotation around its cell body. We infer that the flagellar filament rotates with a transient surface contact, similarly to a screw that is turned into a tapped hole. This will create the force required for propulsion when the filament engages with like the surface roughness. The screwlike motion in flagellated bacteria might be developed as a strategy to move over surfaces without getting stuck and as such be used to escape from environments like the gut with the mucous matrix. 
In the Burkholderia symbiont, we directly visualized the transition of the motility mode from a normal motion to wrapping motion driven by flagellar switching; however, the structural key for the wrapping motion remains unclear. In the very near future, the microscopic measurements in conjunction with mutagenesis into the filament and/or hook will uncover the molecular mechanism(s) of this unique wrapping motion in the Burkholderia symbiont. In addition, Ohbayashi et al. [11] reported that the mutant cheA_AM3 can pass through constricted regions and shows $100 \%$ infection rate in the bean bug, despite no wrapping motion is visible in this mutant (Supplementary Movie 9). We speculate that the wrapping motion is not essential for the penetration behavior, but could influence the penetration efficiency of the Burkholderia symbiont and its transit time in the mucous-filled narrow passage of the constricted region. Therefore the wrapping motion might be relevant to overcome potential competitors. To test this hypothesis, a high-resolution live-imaging of penetrating cells of wild type and AM3 would need to be developed.

After submission of this paper, Kuhn et al., report the spiral-like motion of another bacterium, Shewanella putrefaciens CN-32 [37]. The authors report a similar observation of the left-handed flagellum that eventually wraps around the cell body by switching from $\mathrm{CCW}$ to CW direction. And these cells also shows a screw-like motion driven by $\mathrm{CW}$ rotation of a flagellar filament. The authors claim that this spiral-like motion is important for the interaction between the flagellum and surface, and therefore allows for cellular escape from the complex environments and uneven surfaces. Based on a numerical calculation, they proposed that the instability-driven flagellar polymorphism that the left-handed flagellum is stretched, bended and then transited into the perversions state (the left- and right-handed helix) when increasing the torque on the cell by the $\mathrm{CW}$ rotation, and then, the helix pull toward the cell and wrap its cell body induced by the flagellar switching.

From our results and the Kuhn's finding, we speculate that phylogenetically distinct bacteria share this unique wrapping motion of the polar flagella. Since polar flagella have been reported in diverse bacterial species, this type of wrapping motility may not be restricted to symbiotic bacteria but may be found in other non-symbiotic bacteria such as Pseudomonas putida and Pseudomonas fluorescence, which have a similar swimming feature with reversals $(\theta \sim$ $180^{\circ}$ ) accompanied by rapid changes in speed [14, 15]. The biological functions of this unique motion are probably diverse, and future studies will reveal similarity and differences of flagellar motion in symbiotic and non-symbiotic bacteria, which may contribute the infection specificity and efficiency of symbiotic bacteria.
Acknowledgements The authors thank R. Kamiya and T. E.F. Quax for preparing the manuscript, and T. Minamino and Y.V. Morimoto for supplying E. coli $\mathrm{K}-12 \mathrm{~W} 3110$. This study was supported in part by a grant from the Funding Program for NextGeneration World-Leading Researchers (no. LR033 to T.N.) from the Japan Society for the Promotion of Science, by a Grant-in-Aid for Scientific Research on Innovative Areas "Harmonized Supramolecular Motility Machinery and Its Diversity" (to T.N.) and "Fluctuation \& Structure" (no.26103527 to T.N.) from the Ministry of Education, Culture, Sports, Science, and Technology of Japan, and the Ministry of Education, Culture, Sports, Science and Technology (MEXT) KAKENHI (grant number $15 \mathrm{H} 05638$ for Y. Kikuchi). Y. Kinosita was the recipient of a JSPS Fellowship for Japan Junior Scientists (15J12274) and Postdoctral Fellowship for Research Abroad.

Author contributions Y Kinosita, Y Kikuchi, DN, and TN designed the research; Y. Kinosita performed the research; NM developed a framework for analyzing a reorientation event, Y Kinosita and TN constructed the optical setup and microscope; Y Kinosita, Y Kikuchi and $\mathrm{TN}$ wrote the paper.

\section{Compliance with ethical standards}

Conflict of interest The authors declare that they have no competing interests.

\section{References}

1 Berg HC. The rotary motor of bacterial flagella. Annu Rev Biochem 2003;72:19-54.

2 Turner L, Ryu WS, Berg HC. Real-time imaging of fluorescent flagellar filaments. J Bacteriol 2000;182:2793-801.

3 Berg HC, Brown DA. Chemotaxis in Escherichia coli analysed by three-dimensional tracking. Nature 1972;239:500-4.

4 Son K, Guasto JS, Stocker R. Bacteria can exploit a flagellar buckling instability to change direction. Nat Phys 2013;9:494-8.

5 Xie L, Altindal T, Chattopadhyay S, Wu XL. Bacterial flagellum as a propeller and as a rudder for efficient chemotaxis. Proc Natl Acad Sci USA 2011;108:2246-51.

6 Armitage JP, Schmitt R. Bacterial chemotaxis: Rhodobacter sphaeroides and Sinorhizobium meliloti-variations on a theme? Microbiology 1997;143(Pt 12):3671-82.

7 Scharf B. Real-time imaging of fluorescent flagellar filaments of Rhizobium lupini H13-3: flagellar rotation and $\mathrm{pH}$-induced polymorphic transitions. J Bacteriol 2002;184:5979-86.

8 Chaban B, Hughes HV, Beeby M. The flagellum in bacterial pathogens: for motility and a whole lot more. Semin Cell Dev Biol 2015;46:91-103.

9 Nyholm SV, Mcfall-Ngai MJ. The winnowing: establishing the squid-vibrio symbiosis. Nat Rev Microbiol 2004;2:632-42.

10 Takeshita K, Kikuchi Y. Riptortus pedestris and Burkholderia symbiont: an ideal model system for insect-microbe symbiotic associations. Res Microbiol 2017;168:175-87.

11 Ohbayashi T, Takeshita K, Kitagawa W, Nikoh N, Koga R, Meng $\mathrm{XY}$, et al. Insect's intestinal organ for symbiont sorting. Proc Natl Acad Sci USA 2015;112:E5179-88.

12 Kinosita Y, Nakane D, Sugawa M, Masaike T, Mizutani K, Miyata $\mathrm{M}$, et al. Unitary step of gliding machinery in Mycoplasma mobile. Proc Natl Acad Sci USA 2014;111:8601-6.

13 Kinosita Y, Uchida N, Nakane D, Nishizaka T. Direct observation of rotation and steps of the archaellum in the swimming halophilic archaeon Halobacterium salinarum. Nat Microbiol 2016;1:16148. 
14 Ping L, Birkenbeil J, Monajembashi S. Swimming behavior of the monotrichous bacterium Pseudomonas fluorescens SBW25. FEMS Microbiol Ecol 2013;86:36-44.

15 Theves M, Taktikos J, Zaburdaev V, Stark H, Beta C. A bacterial swimmer with two alternating speeds of propagation. Biophys $\mathrm{J}$ 2013;105:1915-24.

16 Kamiya R, Hotani H, Asakura S. Polymorphic transition in bacterial flagella. Symp Soc Exp Biol 1982;35:53-76.

17 Lai SK, Wang YY, Wirtz D, Hanes J. Micro- and macrorheology of mucus. Adv Drug Deliv Rev 2009;61:86-100.

18 Chen X, Berg HC. Torque-speed relationship of the flagellar rotary motor of Escherichia coli. Biophys J 2000;78:1036-41.

19 Magariyama Y, Sugiyama S, Kudo S. Bacterial swimming speed and rotation rate of bundled flagella. FEMS Microbiol Lett 2001;199:125-9.

20 Berg HC, Turner L. Movement of microorganisms in viscous environments. Nature 1979;278:349-51.

21 Darnton NC, Berg HC. Force-extension measurements on bacterial flagella: triggering polymorphic transformations. Biophys $\mathrm{J}$ 2007;92:2230-6.

22 Nakane D, Sato K, Wada H, Mcbride MJ, Nakayama K. Helical flow of surface protein required for bacterial gliding motility. Proc Natl Acad Sci USA 2013;110:11145-50.

23 Mariconda S, Wang Q, Harshey RM. A mechanical role for the chemotaxis system in swarming motility. Mol Microbiol 2006;60:1590-602.

24 Bartlett TM, Bratton BP, Duvshani A, Miguel A, Sheng Y, Martin $\mathrm{NR}$, et al. A Periplasmic polymer curves Vibrio cholerae and promotes pathogenesis . Cell. 2017;168:172-85e15.

25 O'Shea TM, Deloney-Marino CR, Shibata S, Aizawa S, Wolfe AJ, VISICK KL. Magnesium promotes flagellation of Vibrio fischeri. J Bacteriol 2005;187:2058-65.

26 Davidson SK, Stahl DA. Selective recruitment of bacteria during embryogenesis of an earthworm. ISME J 2008;2:510-8.

27 Dulla GF, Go RA, Stahl DA, Davidson SK. Verminephrobacter eiseniae type IV pili and flagella are required to colonize earthworm nephridia. ISME J 2012;6:1166-75.
28 Kim JK, Lee HJ, Kikuchi Y, Kitagawa W, Nikoh N, Fukatsu T, et al. Bacterial cell wall synthesis gene uppP is required for Burkholderia colonization of the Stinkbug Gut. Appl Environ Microbiol 2013a;79:4879-86.

29 Kim JK, Park HY, Lee BL. The symbiotic role of O-antigen of Burkholderia symbiont in association with host Riptortus pedestris. Dev Comp Immunol 2016;60:202-8.

30 Kim JK, Son DW, Kim CH, Cho JH, Marchetti R, Silipo A, et al. Insect gut symbiont susceptibility to host antimicrobial peptides caused by alteration of the bacterial cell envelope. J Biol Chem 2015;290:21042-53.

31 Kim JK, Jang HA, Won YJ, Kikuchi Y, Han SH, Kim CH, et al. Purine biosynthesis-deficient Burkholderia mutants are incapable of symbiotic accommodation in the stinkbug. ISME J 2014;8:552-63.

32 Kim JK, Won YJ, Nikoh N, Nakayama H, Han SH, Kikuchi Y, et al. Polyester synthesis genes associated with stress resistance are involved in an insect-bacterium symbiosis. Proc Natl Acad Sci USA 2013b;110:E2381-9.

33 Ruby EG. Symbiotic conversations are revealed under genetic interrogation. Nat Rev Microbiol 2008;6:752-62.

34 Visick KL, Foster J, Doino J, Mcfall-Ngai M, Ruby EG. Vibrio fischeri lux genes play an important role in colonization and development of the host light organ. J Bacteriol 2000;182:4578-86.

35 Wang Y, Dunn AK, Wilneff J, Mcfall-Ngai MJ, Spiro S, Ruby EG. Vibrio fischeri flavohaemoglobin protects against nitric oxide during initiation of the squid-vibrio symbiosis. Mol Microbiol 2010;78:903-15.

36 Davidson SK, Koropatnick TA, Kossmehl R, Sycuro L, McfallNgai MJ. NO means 'yes' in the squid-vibrio symbiosis: nitric oxide (NO) during the initial stages of a beneficial association. Cell Microbiol 2004;6:1139-51.

37 Kuhn MJ, Schmidt FK, Eckhardt B, Thormann KM. Bacteria exploit a polymorphic instability of the flagellar filament to escape from traps. Proc Natl Acad Sci USA 2017;114:6340-5. 